\title{
Role and mechanism of nursing cooperation and tetramethylpyrazine application in post-operative pain in patients undergoing total knee arthroplasty
}

\author{
CHANG LIU ${ }^{1}$, RUI LIU ${ }^{2}$, MEILING TANG ${ }^{1}$, XINXIN YANG ${ }^{1}$ and XIUPING GONG ${ }^{3}$ \\ ${ }^{1}$ Surgery Center, The First Affiliated Hospital of Harbin Medical University, Harbin, Heilongjiang 150001; \\ ${ }^{2}$ Department of Hyperbaric Oxygen Therapy, Harbin Children's Hospital, Harbin, Heilongjiang 150010; \\ ${ }^{3}$ Department of Anesthesiology, The First Affiliated Hospital of Harbin Medical University, Harbin, \\ Heilongjiang 150001, P.R. China
}

Received June 1, 2016; Accepted November 22, 2017

DOI: 10.3892/etm.2019.7203

\begin{abstract}
The aim of the present study was to investigate the effect of nursing cooperation on the post-operative complication of pain following total knee arthroplasty (TKA) and to explore the effects of tetramethylpyrazine (TMP) application on post-TKA pain. A total of 26 patients who received TKA between June 2014 and March 2016 were enrolled in this study. Nursing cooperation was provided to the patients during the TKA surgery, and pain was evaluated based on the visual analog scale (VAS). In addition, 40 male Sprague Dawley rats were used for the TKA model construction. The rats were randomly separated into 4 groups (sham, TKA, TKA+TMP and TKA+TMP+Interferon $\gamma$ ). Pain tolerance in rats was evaluated by mechanical stimulation. Inflammatory cytokine levels in TKA rat tissue were detected using ELISA. mRNA and protein expression of Janus kinase/signal transducer and activator of transcription 3 (JAK/STAT3) was detected using reverse transcription-polymerase chain reaction and western blot analysis, respectively. The results indicated that nursing cooperation serves a critical function during TKA and was associated with a lower level of pain compared with the control $(\mathrm{P}<0.05)$. Furthermore, TMP treatment reduced the level of inflammatory cytokines in the rat tissues, including interleukin (IL)-6, IL-10 and tumor necrosis factor- $\alpha$ in post-TKA $(\mathrm{P}<0.01)$. TMP was indicated to alleviate pain in post-TKA through suppressing the JAK/STAT3 signaling pathway. The results of the present
\end{abstract}

Correspondence to: Professor Xiuping Gong, Department of Anesthesiology, The First Affiliated Hospital of Harbin Medical University, 23 Youzheng Road, Harbin, Heilongjiang 150001, P.R. China

E-mail: xiupinggong717@126.com

Key words: total knee arthroplasty, nursing cooperation, tetramethylpyrazine, pain, Janus kinase/signal transducer and activator of transcription 3 study suggest that nursing cooperation is critical to TKA, and TMP may alleviate post-TKA pain through inhibiting the JAK/STAT3 signaling pathway.

\section{Introduction}

Total knee arthroplasty (TKA) has become one of the most effective surgery methods for patients with osteoarticular diseases (1). Pain, hypoxemia and vein thrombosis are the most common complications for patients in post-TKA, which can severely affect patient quality of life (2-4). The factors involved in the complications of TKA are diverse, including the intraoperative state of patients, anesthesia application or nursing $(5,6)$. Nursing intervention may serve a critical function in the process of TKA, and can alleviate the pain in post-TKA (7). In addition, the complication of pain in post-operative TKA still remains a challenge to patients (8). Hence, it will be beneficial to explore novel nursing methods and to develop effective strategies for alleviating pain in post-TKA.

Tetramethylpyrazine (TMP) is an alkaloid monomer that exists in the roots or stems of the Chinese herbal medicine, Ligusticum wallichii (9). Previous studies have reported that TMP primarily acts as an antioxidant, calcium antagonist, inhibitor of platelet aggregation, promoter of microcirculation and blood clots, and regulator of the immune response $(10,11)$. TMP has been widely used in the treatment of cardiovascular disease $(12,13)$. Furthermore, as an anti-inflammatory factor, TMP serves a critical function in numerous types of disease. For example, TMP was identified to reduce the inflammatory mediators induced by amyloid in rat brain microglia (14), and the expression of hypoxia-inducible factor $1 \alpha$ and tumor necrosis factor (TNF)- $\alpha$ were suppressed by TMP in middle cerebral artery occlusion-induced brain ischemia (15). TMP has also been reported to serve a function in alleviating pain following surgery, such as chronic pelvic pain syndrome and neuropathic pain $(16,17)$. However, the mechanisms by which TMP alleviates pain in post-surgery patients remain unclear.

In the current study, the clinical data of patients who received TKA surgery were collected and the effects of 
nursing cooperation on the degree of pain post-TKA were evaluated. Subsequently, a rat TKA model was established and the potential effects of TMP on the degree of pain were analyzed. A possible molecular mechanism of TMP was also investigated. The present study aimed to illustrate the effect of nursing cooperation on pain degree in patients after TKA and to investigate the potential benefits of TMP for relieving pain in clinical TKA patients.

\section{Materials and methods}

Patient recruitment. A total of 26 patients who received TKA in the Surgery Center of The First Affiliated Hospital of Harbin Medical University (Harbin, China) between June 2014 and March 2016 were enrolled in this study. All the patients provided their informed consent and the study was approved by the Ethics Committee of The first Affiliated Hospital of Harbin Medical University, in accordance with the Declaration of Helsinki.

Nursing cooperation and TKA surgery. For the procedure of TKA surgery (18), a total of 13 patients received nursing cooperation (18) and the surgery was performed as follows: i) Preoperative preparation included visiting patients, talking with patients to understand psychological and physical conditions. Furthermore, the nurse explained the anesthesia, treatment and operation procedure to the patients, and alleviated anxiety. Also, patients were informed that the sounds created by the bone hammer, electric saw or electric drill were normal, and that there was no need to be alarmed. The nurse gained the patients' trust so that good cooperation could be obtained during surgery. ii) Circulating nurse: The effective venous infusion channel and other necessary care were prepared by the circulating nurse. During anesthesia, under the help of the circulating nurse, the tracheal intubation or lumbar hemp joint puncture was performed according to the surgery. Hemostatic start time was recorded at the beginning of surgery. Patient status was closely observed to ensure the transfusion was unobstructed, and to guarantee the strict supervision of aseptic technique. For controls $(n=13)$, patients received TKA surgery and were cared for using conventional nursing protocol (18). Following TKA $(n=13)$, continuous passive motion within $5 \mathrm{sec}$ was applied for patients at 6 , 12,24 and 48 h. Pain was evaluated based on the VAS $(0$, no pain; 10, unbearable sharp pain) (19). 'Rest' indicated patients that did not perform any strenuous physical activity after TAK. 'Sport' indicated patients walked after TAK.

Rat model construction and TMP treatment. This animal experiment was approved by the Animal Care Committee of Harbin Medical University Medical Center (Harbin, China). Sprague Dawley rats $(n=40)$ were purchased from Harbin Medical University Medical Center and bred in the Experimental Animal Center. In order to construct the TKA model, rats were anesthetized with $3.5 \%$ chloral hydrate (for $5 \mathrm{~min}$ ) in oxygen and placed in the supine position. The left knee was then bent by $90^{\circ}$ and firmly supported on either side by the blunt ends of sterectactic ear bars. Then, a $0.5 \mathrm{~cm}$ long skin incision was made over the patellar tendon under sterile conditions. The fascia over the muscle and tendon was scraped away with an elevator, then the lateral side of the tendon was freed from the underlying fascia with the top of a scalpel blade. Then, the tendon was removed laterally by $\sim 3 \mathrm{~mm}$ and held in place with a retractor. Subsequently, a hole (1.4 $\mathrm{mm}$ diameter, $0.5 \mathrm{~mm}$ deep) was generated using a diamond drill bur, in both the femur and tibia, at $2 \mathrm{~mm}$ above and below the knee joint, respectively. Finally, the retractor was removed to allow the patella tendon to return to midline. Then the incision was sutured with nylon and $0.3 \mathrm{ml}$ penicillin was provided at the incision for preventing bacterial infection. Rats were randomly divided into TKA+TMP+Interferon $\gamma$ group, TKA+TMP group, TKA group, and sham group with 10 rats in each group. For the TKA + TMP group (17), the holes were immediately filled with 100 mg/kg TMP (batch no. 09110931; Sigma-Aldrich; Merck KGaA, Darmstadt, Germany) during surgery. The TKA group was administered with normal saline at the same dose. Rats in the sham group only underwent a skin incision. All rats were administered with the same duration of exposure to chloral hydrate. All rats were allowed to recover for $36 \mathrm{~h}$ before behavioral testing.

Pain threshold assay. Mechanical withdrawal threshold (MWT) was evaluated using an electronic pain measuring instrument (von Frey filaments, Stoelting Co., Wood Dale, IL, USA), as previously described (20). Briefly, the rats in each group were acclimated in transparent plastic cages with mesh floors for $30 \mathrm{~min}$. Pressure was applied to the plantar surface of each hind paw from using calibrated electronic von Frey filaments and held for $5 \mathrm{sec}$. The force applied at the time of sharp withdrawal was recorded. MWT was measured in triplicate and the results were averaged.

Enzyme-linked immunosorbent assay (ELISA). Inflammatory cytokine levels in rat serum, including IL-6, IL-10 and TNF- $\alpha$, were assessed using ELISA (21). Briefly, rats were anesthetized halothane (3\% for induction and $1.5 \%$ for maintenance; $40 \mathrm{mg} / \mathrm{kg}$ ) and $0.5 \mathrm{ml}$ trunk blood was collected at $48 \mathrm{~h}$ post-surgery among different groups to avoid any substantial time lag in samples collection. Blood samples were centrifuged at $600 \mathrm{x}$ for $15 \mathrm{~min}$ at $4^{\circ} \mathrm{C}$, and supernatants were stored at $-20^{\circ} \mathrm{C}$ until analysis. Pre-treated microtiter plates were coated with $50 \mu \mathrm{g} / \mathrm{ml}$ calf thymus antibodies [IL-6 (cat. no. D6000B), IL-10 (cat. no. R1000), and TNF- $\alpha$ (cat. no. RTA00); R\&D Systems, Inc., Minneapolis, $\mathrm{MN}, \mathrm{USA}$ ] for $2 \mathrm{~h}$ at $37^{\circ} \mathrm{C}$, and then stored overnight at $4^{\circ} \mathrm{C}$. Then, plates were washed with PBS buffer containing $0.05 \%$ Tween-20 (PBST) 3 times, followed by blocking with $5 \%$ goat serum (Sigma-Aldrich, Merck KGaA, Darmstadt, Germany) in PBST for $1 \mathrm{~h}$. Then, serum samples [diluted at 1:100 in PBST containing $10 \%$ calf serum (Sigma-Aldrich, Merck KGaA) and 5\% goat serum] were incubated with horseradish peroxidase (HRP)-conjugated goat anti-mouse IgG (Sigma-Aldrich, Merck KGaA) for $2 \mathrm{~h}$ at $37^{\circ} \mathrm{C}$. The reaction was blocked with $2 \mathrm{~N} \mathrm{H}_{2} \mathrm{SO}_{4}$ and absorbance was measured using a microplate reader (Bio-Rad Laboratories, Inc., Hercules, CA, USA) at $492 \mathrm{~nm}$.

Reverse transcription-quantitative polymerase chain reaction ( $q P C R)$. Total mRNA from rat serum was 
Table I. VAS pain scores for patients following TKA surgery in two groups.

Time after surgery (h)

\begin{tabular}{|c|c|c|c|c|c|}
\hline \multirow[b]{2}{*}{6} & \multirow[b]{2}{*}{12} & \multicolumn{2}{|c|}{24} & \multicolumn{2}{|c|}{48} \\
\hline & & Rest & Sport & Rest & Sport \\
\hline $3.4 \pm 0.4$ & $4.3 \pm 1.2$ & $2.3 \pm 0.8$ & $4.8 \pm 1.2$ & $1.9 \pm 0.7$ & $2.7 \pm 0.7$ \\
\hline $2.1 \pm 0.3^{\mathrm{a}}$ & $3.1 \pm 0.9^{\mathrm{a}}$ & $1.4 \pm 0.9^{\mathrm{a}}$ & $2.9 \pm 0.8^{\mathrm{a}}$ & $0.7 \pm 0.6^{\mathrm{a}}$ & $1.5 \pm 0.6$ \\
\hline
\end{tabular}

${ }^{\text {aP }}<0.05$ vs. control. 'Rest' means patients did not perform any strenuous activity after TAK. 'Sport' means patients walked after TAK. VAS, visual analogue scale; TAK, total knee arthroplasty.

isolated using TRIzol reagent as previously described (22). cDNA was produced using reverse transcriptase (iScript ${ }^{\mathrm{TM}}$ cDNA Synthesis kit; Bio-Rad Laboratories, Inc.) according to manufacturer's protocol. The expression levels of mRNAs were measured by SYBR Green-based qPCR (SYBR Green Master mix, Thermo Fisher Scientific, Inc.) with the following conditions: $95^{\circ} \mathrm{C}$ for $10 \mathrm{~min}, 40$ cycles of $95^{\circ} \mathrm{C}$ for $30 \mathrm{sec}$ and $58^{\circ} \mathrm{C}$ for $30 \mathrm{sec}$. GAPDH was used as the internal control. Primers used for target amplification were as follows: JAK, sense: 5'-GAAGGAATGGGATTGACGAT-3', antisense: 5'-GCAGCCTGTGTTGTTGTCTT-3'; STAT3, sense: 5'-ACC AGCAGTATAGCCGCTTC-3', antisense: 5'-GCCACAATC CGGGCAATCT-3'; and GAPDH, sense: 5'-TATGATGAT ATCAAGAGGGTAGT-3', anti-sense: 5'-TGTATCCAAACT CATTGTCATAC-3'. Relative expression levels of genes were analyzed using the $2^{-\triangle \Delta C q}$ method (23).

Western blot analysis. Synovial tissues of knee were isolated from rats and lysed with radioimmunoprecipitation assay lysis buffer (Sangon Biotech Co., Ltd., Shanghai, China) containing phenylmethanesufonyl fluoride (Sigma-Aldrich; Merck KGaA, Germany), then centrifuged at 10,500 r/g for $10 \mathrm{~min}$ at $4^{\circ} \mathrm{C}$. The supernatant was collected for the measurement of protein concentration using a BCA protein assay kit (Pierce; Thermo Fisher Scientific, Inc.). For western blotting, $30 \mu \mathrm{g}$ protein per cell lysate was subjected to $12 \%$ sodium dodecylsulfate-polyacrylamide gel electrophoresis, then transferred onto polyvinylidene difluoride (PVDF) membranes. The PVDF membranes were blocked in Tris-buffered saline with Tween-20 (TBST) containing $5 \%$ non-fat milk for $1 \mathrm{~h}$ at room temperature. The membranes were incubated with rabbit anti-JAK (cat. no. 702434; 1:100 dilution) and -STAT3 (cat. no. 710077; 1:100 dilution; Invitrogen; Thermo Fisher Scientific, Inc.) and mouse anti-GAPDH antibody (cat. no. MA5-15738-1MG; 1:5,000 dilution; Invitrogen; Thermo Fisher Scientific, Inc.) overnight at $4^{\circ} \mathrm{C}$. Then, the membranes were incubated with HRP-conjugated goat anti-rabbit (cat. no. 62272) or -mouse (cat. no. 35518) secondary antibody (1:1,000 dilution; Invitrogen, Thermo Fisher Scientific, Inc.) at room temperature for $1 \mathrm{~h}$. Finally, the PVDF membranes were washed 3 times with $1 \mathrm{X}$ TBST buffer (10 min/wash). The signals were detected after incubation with a chromogenic substrate using the enhanced chemiluminescence reagent kit (EMD Millipore, Bedford, MA, USA). GAPDH served as the internal control. Rats treated with $10 \mathrm{ng} / \mathrm{ml}$ interferon (IFN) $-\gamma$ for $48 \mathrm{~h}$ (Sigma-Aldrich; Merck KGaA) were introduced as an additional control group.

Statistical analysis. All experiments were independently conducted 3 times. All statistical analyses were performed on GraphPad Prism 5.0 (GraphPad Software, Inc., La Jolla, CA, USA). Data are expressed as the mean \pm standard error of the mean. Independent sample t-test was used to calculate the difference between two groups. One-way analysis of variance with Tukey's post hoc test was used to calculate the difference among groups. $\mathrm{P}<0.05$ was considered to indicate a statistically significant difference.

\section{Results}

Nursing cooperation assists in alleviating pain in post-TKA. To analyze whether nursing cooperation could assist in alleviating pain in post-TKA, the VAS pain scores from patients who received TKA surgery were assessed (Table I). The results indicated that following surgery, the VAS pain score was significantly higher at 6 and $12 \mathrm{~h}$ in the control group ( $\sim 3.4$ at $6 \mathrm{~h} ; \sim 4.3$ at $12 \mathrm{~h}$ ) compared with the nursing cooperation group $(\sim 2.1$ at $6 \mathrm{~h} ; \sim 3.1$ at $12 \mathrm{~h} ; \mathrm{P}<0.05)$. At 24 and $48 \mathrm{~h}$ post-TKA surgery, the VAS pain scores were separated into two subgroups: Rest group and sport group. At each time point, the pain score at rest status was lower compared with sport status. In addition, the VAS pain scores for patients in the nursing cooperation groups were lower compared with the control group at 24 and $48 \mathrm{~h}$ for the rest and sport subgroups (all $\mathrm{P}<0.05$ ). These data suggested that nursing cooperation for TKA may serve a critical function in determining pain in patients following TKA surgery.

TMP alleviates pain in rats undergoing TKA. Pain in post-TKA has become a common issue for patients in the clinic, yet there are few useful strategies for treating this problem. To investigate the effects of TMP on the pain of patients undergoing TKA, a TKA rat model was established. The effect of TMP on rat pain following TKA surgery at 1 , 3, 7 and 14 days was evaluated by the mechanical stimulation method (Fig. 1). MWT decreased over time following surgery in TKA rats compared with the sham group. However, MWT was significantly increased by the application of TMP at 3, 7 and 14 days after surgery $(\mathrm{P}<0.05)$. 
TMP reduces inflammatory cytokine secretion. To analyze the effects of TMP on inflammation in rats following TKA, the levels of cytokines IL-6, IL-10 and TNF- $\alpha$ were detected using ELISA (Fig. 2). The results indicated that the levels of the three cytokines were significantly increased in TKA rats compared with the sham group $(\mathrm{P}<0.01)$. By contrast, their levels were significantly decreased in the TMP-treated group compared with the TKA group $(\mathrm{P}<0.01)$. However, the levels were still higher compared with the sham group. These results suggested that TMP application may reduce inflammatory cytokine secretion to a certain extent following TKA.

TMP suppresses the activation of JAK/STAT3 signaling. It has previously been reported that the JAK/STAT3 signaling pathway is involved in mechanisms of pain, including neuropathic pain (23). Therefore, it was hypothesized that JAK/STAT3 signaling may be involved in the effects of TMP on pain following TKA surgery. Tissues from rats at day 14 post-surgery were used to detect the protein and mRNA expression of JAK and STAT3 (Fig. 3). The results indicated that the mRNA expression level of JAK and STAT3 was significantly decreased in TKA rats at 14 days following application of TMP $(\mathrm{P}<0.01)$. The protein expression of JAK and STAT3 exhibited a similar trend. These results suggested that TMP inhibits JAK/STAT3 signaling.

In addition, IFN- $\gamma$ has been demonstrated to be an activator for JAK/STAT3 signaling $(24,25)$. Therefore, IFN- $\gamma$ treatment $(10 \mathrm{ng} / \mathrm{ml}$ for $48 \mathrm{~h}$ ) was introduced as an additional control group to verify the influence of TMP on JAK/STAT3 signaling. The results indicated that the mRNA expression of JAK and STAT3 was significantly increased in the TKA + TMP + IFN- $\gamma$ group compared with the TKA + TMP group $(\mathrm{P}<0.01)$. The protein expression of JAK and STAT3 exhibited a similar trend. These results supported the proposal that TMP alleviates pain through regulating the JAK/STAT3 signaling pathway.

TMP alleviates TKA pain by regulating the JAK/STAT3 signaling pathway. To further verify the effects of TMP on the JAK/STAT3 signaling pathway, MWT was measured in rats using mechanical stimulation (Fig. 4). As predicted, MWT was markedly decreased in TKA rats compared with the sham group, while this effect was reversed by the application of TMP at days 3, 7 and $14(\mathrm{P}<0.05)$, indicating that TMP suppresses pain caused by TKA. Additionally, the results revealed that the effect of TMP on pain caused by TKA was enhanced by the application of IFN- $\gamma$ at days 3, 7 and $14(\mathrm{P}<0.05)$

\section{Discussion}

Accumulating evidence supports TKA as a treatment strategy for patients who are suffering from osteoarticular diseases $(1,26)$. However, patients who receive TKA often experience complications in post-TKA, including pain $(3,4,27)$. In the present study, the effect of nursing cooperation on the degree of pain experienced by patients in post-TKA was evaluated based on clinical data. Subsequently, a drug treatment was evaluated for the alleviation of pain caused by TKA and its possible molecular mechanism was

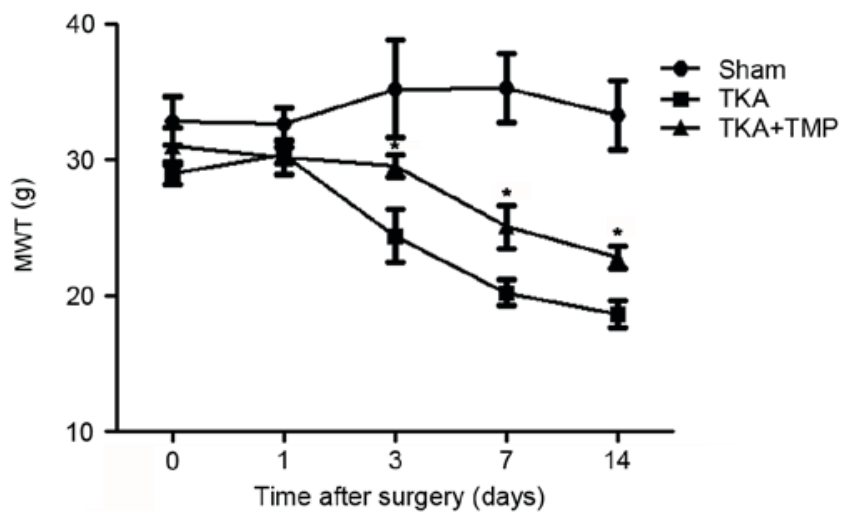

Figure 1. TMP alleviates pain caused by TKA in rats. A mechanical stimulation assay revealed that MWT was markedly decreased in TKA rats compared with the sham group, but it was significantly increased by TMP. ${ }^{*} \mathrm{P}<0.05$ vs. TKA group. TMP, tetramethylpyrazine; TKA, total knee arthroplasty; MWT, mechanical weight threshold.

investigated. Consistent with previous evidence (28), the present results revealed that the VAS pain score for patients in post-TKA who received nursing cooperation during TKA was lower compared with the control group. This indicates a beneficial role of nursing cooperation in alleviating pain in patients following TKA.

Postoperative analgesia is crucial for patients with TKA. Accordingly, in the present study, an anti-inflammatory agent, TMP, was evaluated for treating pain in post-TKA. The major causes for pain in post-TKA are inflammation, nerve pain or mechanical stimulation (29). In accordance with previous studies $(30,31)$, the present data revealed that MWT for rats in post-TKA was significantly decreased compared with the control, and levels of inflammatory cytokines were increased (Figs. 1 and 2). Thompson et al (32) demonstrated that TMP could reduce the level of IL-10 in post-spinal cord injury. Cytokines, including IL- 6 and TNF- $\alpha$, were also suppressed by TMP in other inflammatory conditions, including brain ischemia (15). In the present study, MWT was increased and levels of IL-10, IL- 6 and TNF- $\alpha$ were reduced by TMP treatment. Therefore, it was speculated that TMP could alleviate pain and inhibit inflammation in post-TKA.

Furthermore, the possible molecular mechanism of TMP in alleviating pain was explored. The JAK/STAT3 signaling pathway is not only involved in various biological processes, including cell stress, growth, differentiation and anti-inflammatory effects, but is also involved in the signal transduction of numerous anti-inflammatory factors (33). Molet et al (34) reported that activation of the microglial JAK/STAT3 signaling pathway promoted nerve pain in spinal neurons and astrocytes, suggesting that activation of JAK/STAT3 is an indicator of nerve pain. Similarly, in the present study, the expression of JAK or STAT3 was higher in rats with TKA compared with the control, indicating that JAK/STAT3 signaling is involved in the pain following TKA. The effects of TMP on pain via the JAK/STAT3 signaling pathway have not been fully discussed previously. However, Gao et al (35) demonstrated that TMP inhibited JAK/STAT3 signal transduction in cardiomyocyte hypertrophy. In the present study, the expression of JAK/STAT3 protein was suppressed 
A

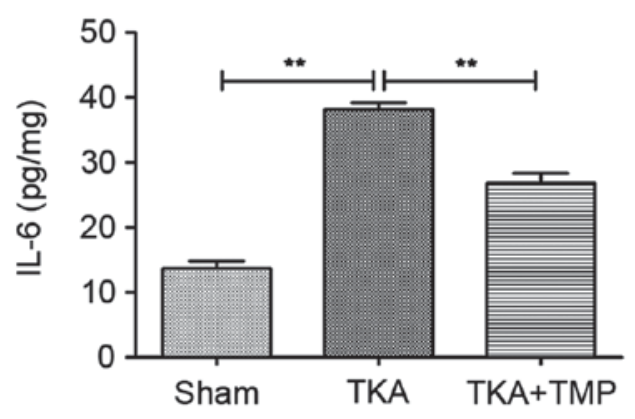

C

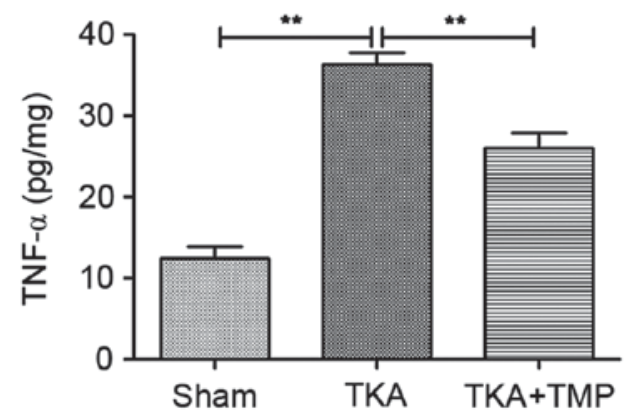

B

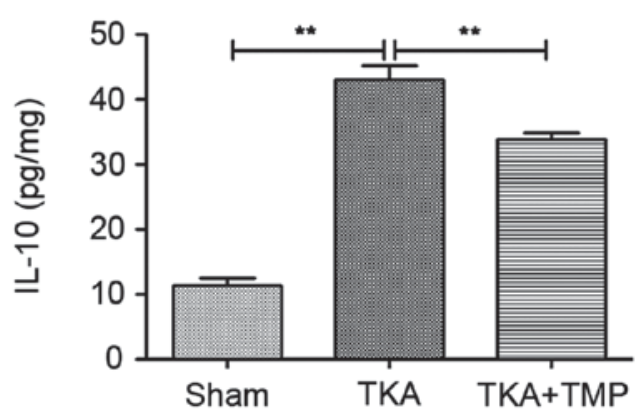

Figure 2. Effect of TMP application on inflammatory cytokine secretion in TKA rats. (A) IL-6 level. (B) IL-10 level. (C) TNF- $\alpha$ level. ${ }^{* *} \mathrm{P}<0.01$. TMP, tetramethylpyrazine; TKA, total knee arthroplasty; IL, interleukin; TNF, tumor necrosis factor.

A

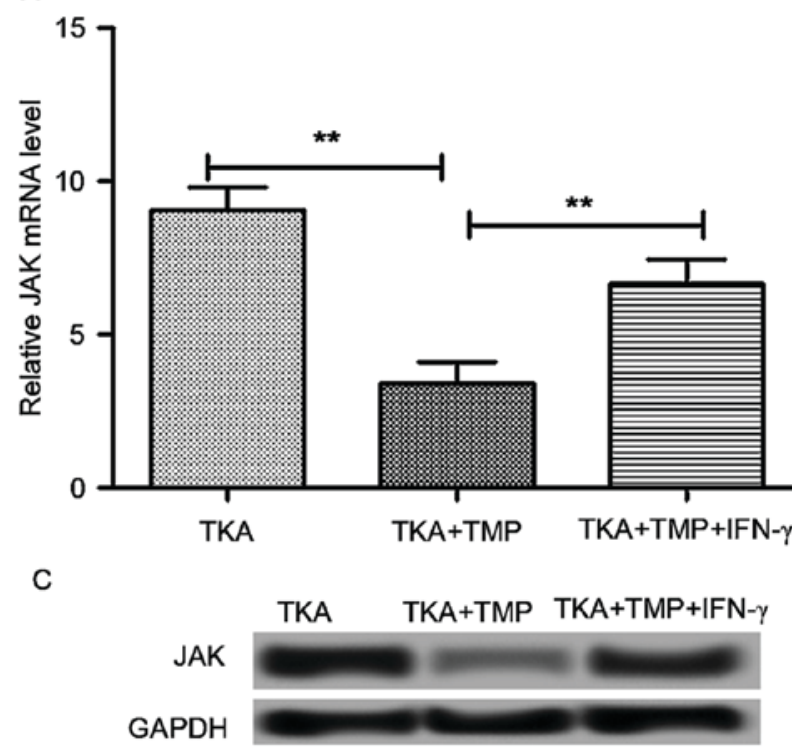

B

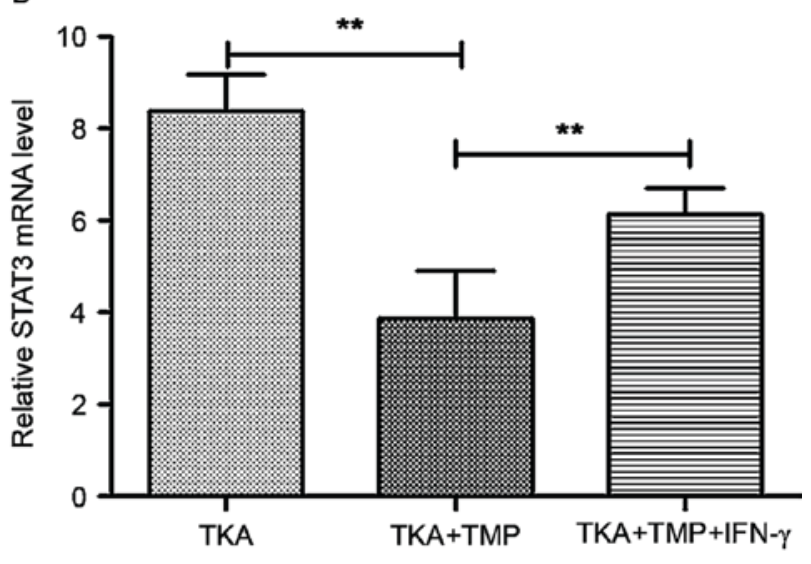

$\mathrm{D}$

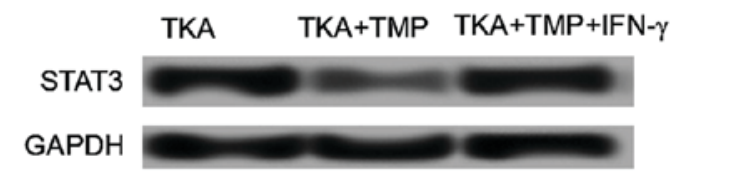

Figure 3. TMP suppresses the JAK/STAT3 signaling pathway. (A) mRNA expression of JAK. (B) mRNA expression of STAT3. (C) Protein expression of JAK. (D) Protein expression of STAT3. ${ }^{* *} \mathrm{P}<0.01$. TMP, tetramethylpyrazine; TKA, total knee arthroplasty; JAK, Janus kinase; STAT3, signal transducer and activator of transcription 3 ; IFN, interferon.

by the application of TMP, implying that TMP alleviates pain in post-TKA by inhibiting the JAK/STAT3 signaling pathway. In addition, IFN- $\gamma$ is an inducer of JAK/STAT3 signaling, and its application can result in inflammation or pain occurrence (36). In the present study, application of IFN- $\gamma$ increased MWT in post-TKA rats, which indicated an enhanced effect of TMP on JAK/STAT3 signaling for alleviating pain.
In summary, the data presented in the present study suggests that nursing cooperation may serve a critical function during the process of TKA and may reduce the degree of pain experienced by patients following TKA. In addition, the present study suggests that TMP may alleviate pain following TKA via suppressing the JAK/STAT3 signaling pathway. The current study highlights the importance of nursing cooperation during TKA, and a possible application of TMP for 


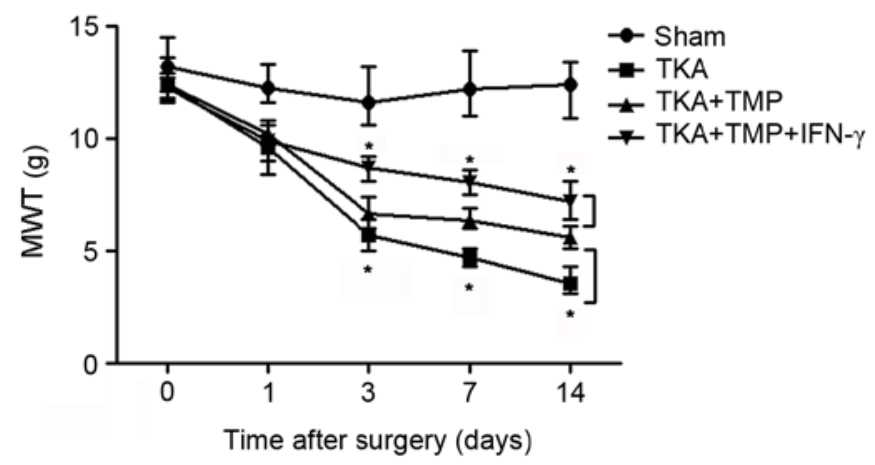

Figure 4. TMP alleviates pain caused by TKA via regulating the JAK/STAT3 signaling pathway. MWT was markedly decreased in rats with TKA compared with the sham group, but TMP treatment increased MWT significantly compared with the TKA group. However, the activator of JAK/STAT3 signaling, IFN- $\gamma$, enhanced the effect of TMP on MWT. "P<0.05. TMP, tetramethylpyrazine; TKA, total knee arthroplasty; MWT, mechanical weight threshold; JAK, Janus kinase; STAT3, signal transducer and activator of transcription 3; IFN, interferon.

the clinical treatment of pain in post-TKA. However, further studies are required to explore the detailed mechanism of TMP in alleviating pain for patients following TKA.

\section{References}

1. Wakabayashi H, Takigawa S, Hasegawa M, Kakimoto T, Yoshida K and Sudo A: Polyarticular late infection of total joint arthroplasties in a patient with rheumatoid arthritis treated with anti-interleukin-6 therapy. Rheumatology (Oxford) 53: 1150-1151, 2014.

2. Eskander MS, Mcphee E, Eskander JP, Nascimento R, Mccormick JJ, Hao S, Shepro D and Johnson K: A left knee wound complication by non-Hodgkins lymphoma in bilateral total knee arthroplasties. Arch Orthop Trauma Surg 128: 1387-1390, 2008.

3. Belmont PJ Jr, Goodman GP, Waterman BR, Schoenfeld AJ and Bader JO: Thirty-day postoperative complications and mortality following total knee arthroplasty: Incidence and risk factors among a national sample of 15,321 patients. J Bone Joint Surg Am 96: 20-26, 2014.

4. Pugely AJ, Martin CT, Gao Y, Mendoza-Lattes S and Callaghan JJ: Differences in short-term complications between spinal and general anesthesia for primary total knee arthroplasty. J Bone Joint Surg Am 95: 193-199, 2013.

5. Nuelle DG and Mann K: Minimal incision protocols for anesthesia, pain management, and physical therapy with standard incisions in hip and knee arthroplasties: The effect on early outcomes. J Arthroplasty 22: 20-25, 2007.

6. Allepuz A, Espallargues M, Moharra M, Comas M and Pons JM; Research Group on Support Instruments-IRYSS Network: Prioritisation of patients on waiting lists for hip and knee arthroplasties and cataract surgery: Instruments validation. BMC Health Serv Res 8: 75, 2008.

7. Hejblum G, Atsou K, Dautzenberg B and Chouaid C: Cost-benefit analysis of a simulated institution-based preoperative smoking cessation intervention in patients undergoing total hip and knee arthroplasties in France. Chest 135: 477-483, 2009.

8. Sharkey PF, Lichstein PM, Chao S, Tokarski AT and Parvizi J: Why are total knee arthroplasties failing today-has anything changed after 10 years? J Arthroplasty 29: 1774-1778, 2014.

9. Ni X, Wong SL, Wong CM, Lau CW, Shi X, Cai Y and Huang Y: Tetramethylpyrazine protects against hydrogen peroxide-provoked endothelial dysfunction in isolated rat aortic rings: Implications for antioxidant therapy of vascular diseases. Evid Based Complement Alternat Med 2014: 627181, 2014.

10. Gaetano GD, Bottecchia D and Vermylen J: Retraction of reptilase-clots in the presence of agents inducing or inhibiting the platelet adhesion-aggregation reaction. Thromb Res 2: 71-84, 1973.
11. Sarkar S, Freedman J and Varghese S: Methods and kits for sustaining eNOS activity to inhibit platelet aggregation, and clot retraction, and promote fibrinolysis. Journal, 2011.

12. Wang GF, Shi CG, Sun MZ, Wang L, Wu SX, Wang HF, Xu ZQ and Chen DM: Tetramethylpyrazine attenuates atherosclerosis development and protects endothelial cells from ox-LDL. Cardiovasc Drugs Ther 27: 199-210, 2013.

13. Li WS, Yang C, Shan L, Zhang Z, Wang Y, Kwan YW, Lee SM, Hoi MP, Chan SW, Cheung AC, et al: Relaxation effect of a novel Danshensu/tetramethylpyrazine derivative on rat mesenteric arteries. Eur J Pharmacol 761: 153-160, 2015.

14. Kim M, Kim SO, Lee M, Lee JH, Jung WS, Moon SK, Kim YS, Cho KH, Ko CN and Lee EH: Tetramethylpyrazine, a natural alkaloid, attenuates pro-inflammatory mediators induced by amyloid $\beta$ and interferon- $\gamma$ in rat brain microglia. Eur J Pharmacol 740: 504-511, 2014.

15. Chang Y, Hsiao G, Chen SH, Chen YC, Lin JH, Lin KH, Chou DS and Sheu JR: Tetramethylpyrazine suppresses HIF-1alpha, TNF-alpha, and activated caspase-3 expression in middle cerebral artery occlusion-induced brain ischemia in rats. Acta Pharmacol Sin 28: 327-333, 2007.

16. Sanders HR and Albitar M: TMPRSS2 for the diagnosis of prostate disease. Journal, 2013.

17. Leng YF, Gao XM, Wang SX and Xing YH: Effects of tetramethylpyrazine on neuronal apoptosis in the superficial dorsal horn in a rat model of neuropathic pain. Am J Chin Med 40: 1229-1239, 2012.

18. Macario A, Schilling P, Rubio R and Goodman S: Economics of one-stage versus two-stage bilateral total knee arthroplasties. Clin Orthop Relat Res 414: 149-156, 2003.

19. Kelly AM: The minimum clinically significant difference in visual analogue scale pain score does not differ with severity of pain. Emerg Med J 18: 205-207, 2001.

20. Vivancos GG, Verri WA Jr, Cunha TM, Schivo IR, Parada CA, Cunha FQ and Ferreira SH: An electronic pressure-meter nociception paw test for rats. Braz J Med Biol Res 37: 391-399, 2004.

21. Li P, Xie TB, Yi ZR, Wei LL, Wang DM, Zhang JY, Ou X, Chen $\mathrm{F}$ and Luo X: Research on detection results of aflatoxin M1 enzyme-linked immune sorbent assay detection kit. J Food Saf Qual 6: 2297-2302, 2015.

22. Rancourt MF, Kemp KA, Plamondon SM, Kim PR and Dervin GF: Unicompartmental knee arthroplasties revised to total knee arthroplasties compared with primary total knee arthroplasties. J Arthroplasty 27 (8 Suppl): S106-S110, 2012.

23. Dominguez E, Rivat C, Pommier B, Mauborgne A and Pohl M: JAK/STAT3 pathway is activated in spinal cord microglia after peripheral nerve injury and contributes to neuropathic pain development in rat. J Neurochem 107: 50-60, 2008.

24. Takaoka A, Tanaka N, Mitani Y, Miyazaki T, Fujii H, Sato M, Kovarik P, Decker T, Schlessinger J and Taniguchi T: Protein tyrosine kinase Pyk2 mediates the Jak-dependent activation of MAPK and Stat 1 in IFN-gamma, but not IFN-alpha, signaling. EMBO J 18: 2480-2488, 1999.

25. Kim J, Yoon Y, Jeoung D, Kim YM and Choe J: Interferon- $\gamma$ stimulates human follicular dendritic cell-like cells to produce prostaglandins via the JAK-STAT pathway. Mol Immunol 66: 189-196, 2015.

26. Caplan N and Kader DF: A comparison of four models of total knee-replacement prostheses. In: Banaszkiewicz P and Kader D (eds) Classic Papers in Orthopaedics. Springer London, pp169-171, 2014.

27. Cobb JP: Patient safety after partial and total knee replacement. Lancet 384: 1405-1407, 2014.

28. Akyol O, Karayurt O and Salmond S: Experiences of pain and satisfaction with pain management in patients undergoing total knee replacement. Orthop Nurs 28: 79-85, 2009.

29. Tanavalee A, Honsawek S, Rojpornpradit T, Sakdinakiattikoon M and Ngarmukos S: Inflammation related to synovectomy during total knee replacement in patients with primary osteoarthritis: A prospective, randomised study. J Bone Joint Surg Br 93: 1065-1070, 2011.

30. Balandraud N, Meynard JB, Auger I, Sovran H, Mugnier B, Reviron D, Roudier J and Roudier C: Epstein-Barr virus load follow up in rheumatoid arthritis patients treated with TNF- $\alpha$ inhibitors. Arth Res Ther 6: 91, 2004.

31. Berbari E, Mabry T, Tsaras G, Spangehl M, Erwin PJ, Murad MH, Steckelberg J and Osmon D: Inflammatory blood laboratory levels as markers of prosthetic joint infection: A systematic review and meta-analysis. J Bone Joint Surg Am 92: 2102-2109, 2010. 
32. Thompson CD, Zurko JC, Hanna BF, Hellenbrand DJ and Hanna A: The therapeutic role of interleukin-10 after spinal cord injury. J Neurotrauma 30: 1311-1324, 2013.

33. Lee C, Lim HK, Sakong J, Lee YS, Kim JR and Baek SH: Janus kinase-signal transducer and activator of transcription mediates phosphatidic acid-induced interleukin (IL)-1beta and IL-6 production. Mol Pharmacol 69: 1041-1047, 2006.

34. Molet J, Mauborgne A, Diallo M, Armand V, Geny D, Villanueva L, Boucher Y and Pohl M: Microglial Janus kinase/signal transduction and activator of transcription 3 pathway activity directly impacts astrocyte and spinal neuron characteristics. J Neurochem 136: 133-147, 2016.
35. Gao MH, Zhang L, Li B, Ren SR and Zhang B: Effect of tetramethylpyrazine on JAK-STAT signal transduction in cardiomyocyte hypertrophy. Xi Bao Yu Fen Zi Mian Yi Xue Za Zhi 27: 519-521, 524, 2011 (In Chinese).

36. Delgado M: Inhibition of interferon (IFN) gamma-induced Jak-STAT1 activation in microglia by vasoactive intestinal peptide: Inhibitory effect on CD40, IFN-induced protein-10, and inducible nitric-oxide synthase expression. J Biol Chem 278 27620-27629, 2003. 